\title{
Ein Datenschutzrecht für das 21. Jahrhundert
}

Lange Zeit herrschte Stillstand bei der Modernisierung des Datenschutzrechts. Obwohl allgemein Einigkeit darüber bestand, die in ihrer Grundstruktur noch aus der Frühzeit des Datenschutzes stammenden Regelungen seien dem digitalen Zeitalter nicht mehr angemessen und bedürften einer umfassenden Reform, wurden aus dieser Einsicht keinerlei Konsequenzen gezogen. So öffnete sich die Schere zwischen den realen Anforderungen an einen wirksamen Datenschutz und dem zur Verfügung stehenden datenschutzrechtlichen Instrumentarium immer mehr, erst recht, nachdem die Terroranschläge des 11. Septembers 2001 auch die Vorbereitungen zur sogenannten 2. Stufe der Novellierung des BDSG abrupt beendet hatten. Bewegung kam in die Reformdebatte erst wieder in jüngster Zeit, als zahlreiche Datenschutzskandale auf der einen Seite und grundlegende Gerichtsentscheidungen auf der anderen den akuten Handlungsbedarf jedermann vor Augen führten. Als erste Reaktion hat der Deutsche Bundestag im letzten Jahr gleich zwei Novellen zur Änderung des BDSG beschlossen, die aber eher punktuell die Vorschriften zu Scoreverfahren und Auskunfteien und zum Adresshandel zu Zwecken der Werbung reformiert haben. Eine grundlegende Modernisierung des Datenschutzrechts war damit nicht verbunden, obwohl insbesondere die Informationspflicht bei Datenschutzpannen, die Stärkung der Rechte der Aufsichtsbehörden und die überarbeiteten Bestimmungen zur Auftragsdatenverarbeitung in die richtige Richtung gehen. Es blieb aber im Wesentlichen bei Reparaturarbeiten am geltenden Recht.

Das Thema bleibt indes auf der Tagesordnung: So soll noch in diesem Jahr ein eigenes umfangreiches Kapitel zum Beschäftigtendatenschutz ins BDSG integriert werden. Auch der Datenschutz im Internet wird breit diskutiert und die Debatte hat bereits zu ersten gesetzgeberischen Initiativen geführt. Es besteht kein Zweifel, dass weitere Schritte in dieser Richtung beabsichtigt sind. Die Koalitionsvereinbarung der aktuellen Bundesregierung sieht eine Stiftung Datenschutz vor, die u. a. ein Datenschutzaudit entwickeln soll. Auch in diesem Bereich bleibt also Bewegung, nachdem im letzten Jahr das Datenschutzauditgesetz gescheitert war.

All diesen Überlegungen und Aktivitäten ist gemeinsam, dass sie zwar Einzelthemen aufgreifen, aber ein Gesamtkonzept für die Reform des Datenschutzrechts nicht ersetzen können. Deshalb haben die Datenschutzbeauftragten des Bundes und der Länder ein umfassendes und detailliertes Eckpunktepapier erarbeitet und auf ihrer Frühjahrskonferenz am 18. März 2010 in Stuttgart beschlossen. Die Reformüberlegungen werden auch Gegenstand eines Symposiums am 4. Oktober 2010 in Berlin sein.

Die Datenschutzreform muss dem Grundrecht auf informationelle Selbstbestimmung im Zeitalter des Internets und der allgegenwärtigen Informationsverarbeitung wieder Geltung zu verschaffen.

Sowohl die Systematik als auch die Mechanismen zur Durchsetzung des Datenschutzes bedürfen der Modernisierung:

- Die Datenschutzgesetze sollten allgemeingültige datenschutzrechtliche Grundregelungen und verbindliche Mindeststandards festlegen. Ausgehend von diesen Schutzzielen sind sanktionsbewehrte Grundsatznormen zu formulieren, die für alle Formen der Datenverarbeitung gleichermaßen gelten. Dabei sollte ein technikneutraler Ansatz verfolgt werden, der unabhängig vom aktuellen Stand der Technik Schutzziele festlegt, die auch bei technologischen Änderungen uneingeschränkt zur Geltung kommen.

- Die Grundsätze der Datenvermeidung, Datensicherheit und der Kontrolle der Betroffenen über ihre Daten müssen bereits beim Systementwurf berücksichtigt werden (privacy by design). Erforderlich sind auch verbindliche Vorgaben $\mathrm{zu}$ datenschutzfreundlichen Voreinstellungen elektronischer Dienste (privacy by default).

- Das Datenschutzrecht muss dafür sorgen, dass die Datenverarbeitung für die Betroffenen transparenter wird. Außerdem muss die Wahrnehmung der
Datenschutzrechte erleichtert werden. Denn nur ein kritischer und handlungsfähiger Betroffener kann aktiv sein Grundrecht auf informationelle Selbstbestimmung wahrnehmen und nicht nur bloßes Objekt der Datenverarbeitung anderer sein.

- Die Forderung, das Datenschutzrecht internetfähig zu machen, bedeutet zum einen, dass eine unbeobachtete Kommunikation und Nutzung elektronischer Dienste gewährleistet werden muss. Darüber hinaus muss der Betroffene seine Datenschutzrechte auch im Internet durchsetzen können, etwa dadurch, dass ihm technische Möglichkeiten zum Schutz seiner Privatsphäre zur Verfügung stehen.

- Wirtschaft und Verwaltung müssen den Datenschutz als eigenes Anliegen begreifen und für mehr Eigenkontrolle sorgen, etwa durch verbindliche $\mathrm{Da}$ tenschutzkonzepte.

- Die unabhängige Datenschutzaufsicht muss gestärkt und endlich rechtlich und tatsächlich durch ausreichende personelle und finanzielle Mittel in die Lage versetzt werden, ihre Aufgaben zu erfüllen. Hierzu gehören auch wirksamere Sanktionsmöglichkeiten, sowohl bei Straf- und Bußgeldvorschriften als auch im Bereich der Haftung.

Damit ist ein Rahmen vorgegeben, wie eine grundlegende Reform des Datenschutzrechts aussehen könnte, die den Herausforderungen des 21. Jahrhunderts entspricht. Es ist zu hoffen, dass das Eckpunktepapier der Datenschutzbeauftragten des Bundes und der Länder eine breite Diskussion aller Betroffenen und Interessierten anstößt, die zu konkreten gesetzgeberischen Schritten führt. Alle Interessierten sind eingeladen, sich an der Diskussion zu beteiligen, etwa in dem für diesen Zweck eingerichteten elektronischen Diskussionsforum ( $w w w$.datenschutz-forum.bund.de).

Peter Schaar

Der Bundesbeauftragte für den

Datenschutz und die Informationsfreiheit 